\title{
Dynamical process of switch-off in a supertwisted nematic cell
}

\author{
Fuzi Yang ${ }^{\text {a) }}$ and Youmei Dong \\ Key Lab of Organic Optoelectronics \& Molecular Engineering of Ministry of Education, Beijing 100084, \\ China and Liquid Crystal Research Centre, Chemistry Department, Tsinghua University, \\ Beijing 100084, China \\ L. Z. Ruan and J. R. Sambles \\ Thin Film Photonics, School of Physics, University of Exeter, Exeter, EX4 4QL, United Kingdom
}

(Received 22 December 2003; accepted 9 April 2004)

\begin{abstract}
The switch-off dynamics of a $180^{\circ}$ supertwisted nematic (STN) are explored in detail by using a convergent beam system and the fully leaky guided-mode technique. From the dynamical guided-mode data and using the Ericksen-Leslie theory, the director structure in the cell at different times during switch-off is obtained. We have studied the dynamic switch-off from different applied voltages and have found that the relaxation time is independent of the voltage. From fitting the optical data, the effective viscosities are obtained. It appears that two viscosity coefficients, $\gamma$ and $\eta_{1}$, largely control the dynamics of switch-off. From the analysis of the director tilt profile in the cell, it is found that there is no backflow-induced increased tilt at the cell center in the STN cell during the switch-off. From liquid crystal hydrodynamics, this no-backflow dynamic process in the STN cell is well explained. (C) 2004 American Institute of Physics. [DOI: 10.1063/1.1757031]
\end{abstract}

\section{INTRODUCTION}

Although twisted-nematic (TN) cells are very extensively used in liquid crystal (LC) displays, their narrow viewing angle, relatively limited electro-optic response, and slow switching time limit their application. This is particularly true for display devices, which require fast switching and high multiplexibility. In order to overcome these disadvantages in TN cells the supertwisted nematic (STN) device (the director twist angle in the cell is more than $90^{\circ}$ ) was developed and has been widely used in portable, notebook computer and mobile phone displays. This device has a wider viewing angle and faster electro-optic response. Furthermore, due to the slow electro-optical response in the TN cell, the number of lines in the matrix has been greatly restricted in rms multiplexing applications, so that the TN cell is only used in quite low-resolution displays. On the other hand, with the steep electro-optical characteristics in the STN cell, it is possible to achieve a very high multiplexing level. Thus, STN devices are very suitable for high information content displays. In order to fully understand its operation the dynamics of the switching process needs to be explored.

The Ericksen-Leslie theory ${ }^{1-3}$ is the elementary work concerning dynamic effects in nematic LCs. This theory was developed in the 1960s, and is still very widely used, being regarded by most practitioners as the definitive theory. An important approximation was later developed by Berreman ${ }^{4}$ and van Doorn, ${ }^{5}$ which made the theory of more practical utilization. There also exist a number of experimental studies $^{6,7}$ on LC dynamics. One of the most significant experiments confirmed the theoretically predicted "backflow"

\footnotetext{
a) Author to whom correspondence should be addressed; electronic mail: yangfz@mail.tsinghua.edu.cn
}

in a TN cell during switch-off from a strong external electric field. ${ }^{6,7}$ However, almost all the experiments ${ }^{6-8}$ have been based on simple transmission or reflection observations during switching, including those experiments that confirm the predicted backflow. As such, the signals from the LC cell are an integrated response. The details of the director profile in the LC cell cannot be unambiguously obtained, that is, the changes of the director profile in the cell during the switching process cannot be examined in detail. Only recently, using a convergent-beam optical guided-wave technique $e^{9-12}$ have the details of the director profile in the LC cell during the switching process been unambiguously obtained.

Optical waveguide techniques ${ }^{13-15}$ are now well established for accurately determining the director profile in LC cells. Such techniques are based on the excitation of a set of optical guided modes within the LC cell, each being sensitive differently to the director orientation in different spatial regions through the cell. With the implementation of the fully leaky (FL) waveguide geometry ${ }^{16}$ standard commercial cells can be directly investigated using these powerful techniques. The introduction of a convergent-beam system ${ }^{9,10}$ with a CCD array detector further improved the FL technique. In the conventional waveguide study, it takes some time (minutes) to record the guided-mode data over a wide range of incident angles. The convergent-beam technique encompasses simultaneously a wide angle range, so that there is no need to rotate the sample to record the data, and the CCD camera can been used to capture the whole optical response of the cell during the switching process, with submillisecond time resolution. This then allows the direct study of the cell dynamics. Furthermore, if the convergent-beam spot is small enough, for example, down to even a few microns in diameter, this technique may be used to investigate the director structure in single pixels. It has already been 


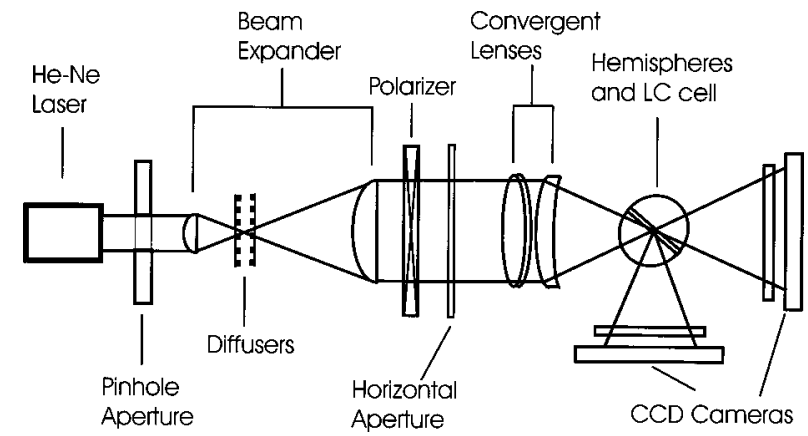

FIG. 1. The experimental setup for the convergent-beam system.

used to investigate the director response in a $90^{\circ} \mathrm{TN}$ cell during both switch-on and switch-off. ${ }^{11,12}$

In this present study, the convergent-beam system is used to investigate the switch-off dynamics of an $180^{\circ} \mathrm{STN}$ cell, filled with a chiral material, for three starting voltages. Using multilayer optics modeling, the director profiles in the cell at different times are analyzed and compared with predictions based on the Ericksen-Leslie theory, yielding thereby viscosity coefficients and a detailed picture of director flow.

\section{EXPERIMENT}

The experimental setup for the convergent-beam system is shown in Fig. 1. The He-Ne laser beam of wavelength $632.8 \mathrm{~nm}$ is collimated into a less-coherent parallel beam of diameter about $5 \mathrm{~cm}$ by using the diffusers and beam expander., ${ }^{9,10}$ This collimated beam passes through a polarizer, a horizontal slit aperture, and a pair of converging lenses, being then focused to a spot at the center of the cell to be studied. The cell is surrounded by two hemispherical prisms that are optically coupled to the cell by matching fluid. The hemispherical prisms and the matching fluid have the same index as the cell substrates. Finally, the reflectivity and transmissivity from the cell are recorded through a polarizer by a CCD camera (DALSA).

The cell structure is the FL geometry. Two low-index glass plates ( $n=1.5170$ at $632.8 \mathrm{~nm}$ ), each coated with a thin (about $50 \mathrm{~nm}$ ) layer of the transparent conductor, indium tin oxide (ITO), and surface aligning layers of rubbed polyimide are assembled together with $6.0-\mu \mathrm{m}$ spacers in between. The angle between the rubbing directions of the upper and lower plates is $0^{\circ}$, forming a left-handed $180^{\circ}$ STN cell with a chiral material. The filling LC material is a mixture of ZLI5100-000 and ZLI-5100-100, to which was also added a small quantity of a dopant ZLI-811 to help the formation of a uniform twisted structure in the cell. The pitch of the material is $14.29 \mu \mathrm{m}$. From polarization microscopy, the director in this STN cell is found to be uniformly aligned, with no visible defects in the cell.

The complete assembly, consisting of the cell, matching fluid, and two glass hemispheres, is placed so that the center of the sample is at the focus of the convergent laser beam. In order to obtain more sensitive data to the director profile in the cell, it is first set such that the rubbing direction at the surface has an angle of about $45^{\circ}$ with the incident plane. We then chose the incident angular region of the FL guided modes from about $60^{\circ}$ up to $78^{\circ}$ by setting the angle between the central axis of the convergent beam and the cell normal to be $\sim 70^{\circ}$ (for details, see Ref. 11). According to the sensitivity and the line transfer rate of the CCD camera, and the details of the optical system, a $0.5 \mathrm{~ms}$ exposure time of the CCD camera was chosen for taking high quality dynamical data. With this exposure time and quite a strong diffuser, a 70 $\mathrm{mW}$ He-Ne laser operating at $632.8 \mathrm{~nm}$ gives data with a good signal-to-noise ratio.

By choice of input polarization setting ( $p$, transverse magnetic, or $s$, transverse electric) and output polarization setting, a variety of angle-dependent signals may be recorded, including the polarization conserving reflectivities $R_{p p}$ and $R_{s s}$, polarization conserving transmissivities $T_{p p}$ and $T_{s s}$, as well as polarization conversion reflectivity $R_{p s}$ and $R_{s p}$ and transmissivity signals $T_{p s}$ and $T_{s p}$. Data are first recorded with no voltage applied to the cell to characterize the cell in its original optical state. Next, ac voltages (10 $\mathrm{kHz}$ ) of $2.8,4.7$, and $6.8 \mathrm{~V}$, are applied to the cell and data recorded in the voltage-on steady-state of the cell. Finally, on switching-off the voltages from $2.8,4.7$, and $6.8 \mathrm{~V}$, to $0 \mathrm{~V}$, the dynamic relaxation of the cell is recorded by synchronizing the CCD camera to capture the data. Figure 2 shows how the guided mode signal $T_{s s}$ during switch-off from different applied voltages varies with time. These figures show a dramatic change in the guided-mode structure during switch-off.

\section{RESULTS AND DISCUSSION}

Firstly, it is clear from Fig. 2 that the relaxation times in the cell for switch-off from different applied voltages are very similar. This is because there is no applied field acting on the cell during this process, the relaxation depends only on the material parameters and the cell structure. In this cell, the total recovery time is about $60 \mathrm{~ms}$, which is much faster than that in a TN cell. For a TN cell with the same thickness, the recovery time of switch-off from an electric field is about 120 ms. $^{11}$

Using multilayer optical theory ${ }^{17}$ to fit the reflection and transmission data the full director profile and the material parameters of the different layers in the cell are obtained. For no applied field, the optical parameters of the different layers at $632.8 \mathrm{~nm}$ are first found as follows. The ITO layers each have a thickness of $50 \mathrm{~nm}$ and an optical permittivity of $\varepsilon$ $=3.3000+i 0.12$; while the polyimide layers have anisotropic optical permittivities $\varepsilon_{\perp}=2.2200+i 0.001, \quad \varepsilon_{\|}$ $=2.4000+i 0.001$ with a thickness of $25.0 \mathrm{~nm}$. The optical permittivities of the liquid crystal mixture are $\varepsilon_{\perp}=2.2380$ $+i 0.0005, \varepsilon_{\|}=2.6936+i 0.0001$ with the thickness of the LC layer equal to $5.76 \mu \mathrm{m}$. The director uniformly twists through the whole parallel aligned cell from the top to the bottom through an angle of $180^{\circ}$, with surface tilts of about $1.0^{\circ}$ on both surfaces. After an ac voltage is applied to the cell, the director structure in the cell is substantially altered. Based on the Frank-Oseen elasticity theory, from fitting, the data taken with the applied fields, the values of the elastic constants and the dielectric permittivities of the LC material are determined as follows. The elastic constants $K_{11}, K_{22}$, 


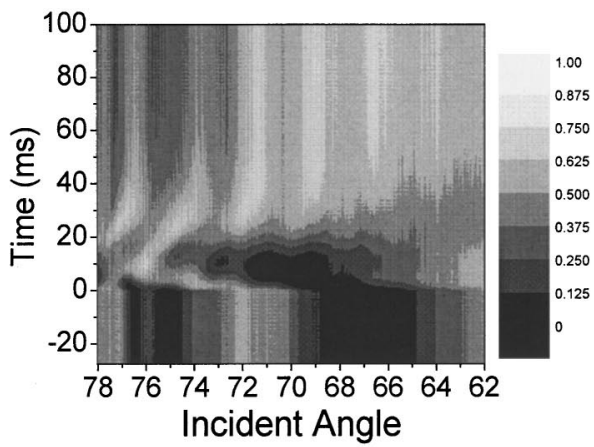

(a)
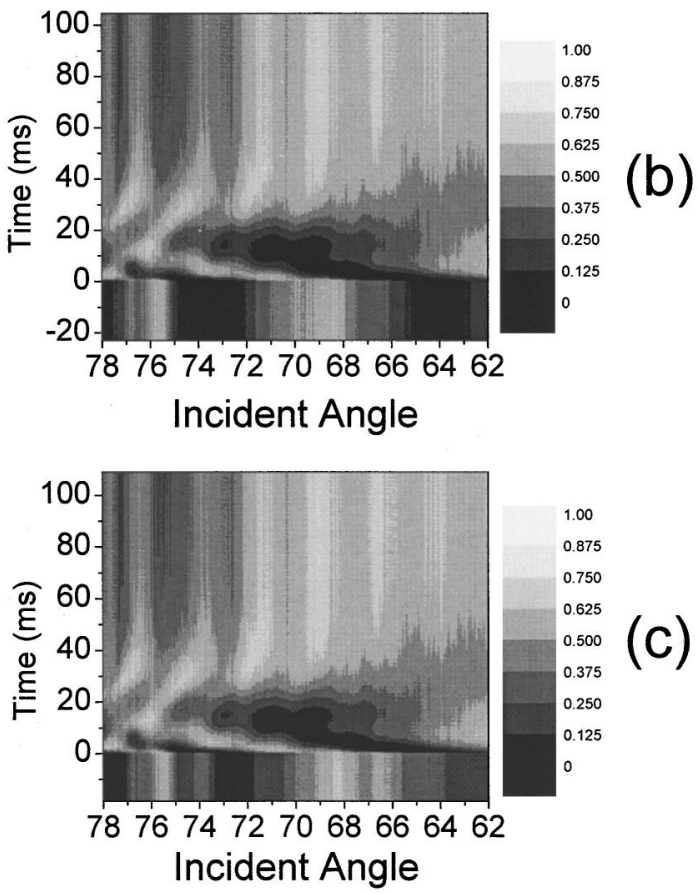

FIG. 2. The dynamic data of $T_{s s}$ after switch-off from (a) 2.8, (b) 4.7, and (c) $6.8 \mathrm{~V}(10 \mathrm{KHz})$.

and $K_{33}$ are 12.84( \pm 0.01$), 7.92( \pm 0.01)$, and $19.00( \pm 0.01)$ $\mathrm{pN}$, respectively; the dielectric permittivities are $\varepsilon_{\perp}=4.5$ $( \pm 0.1), \varepsilon_{\|}=16.0( \pm 0.1)$. Figure 3 shows the director profiles for no applied voltage and with applied voltages of 2.8 , 4.7 , and $6.8 \mathrm{~V}$.

Figure 4 shows the fitted results for an applied voltage of 4.7 V. From Fig. 3 it is clear that at higher applied voltage, the director in the majority of the cell is almost homeotropically aligned, with a very slow linear change of the director twist near both surfaces. Due to the chirality of the LC, the directors at both surfaces of the cell are along the "easy" alignment axes, which are twisted by $180^{\circ}$ from the top to the bottom.

With the static director profile established, attention turns to the main purpose of this study, dynamics in an STN cell during switch-off. By using a modeling program (DIMOS), which is based on the Ericksen-Leslie hydrodynamic theory ${ }^{1-3}$ and uses the approximation of Berreman ${ }^{4}$ and van Doorn, ${ }^{5}$ director profiles are produced. This means that which from multilayer optics model predictions provide fits to the dynamic data. In this modeling, the fluid inertia is ignored and flow is restricted to the plane of the cell. The
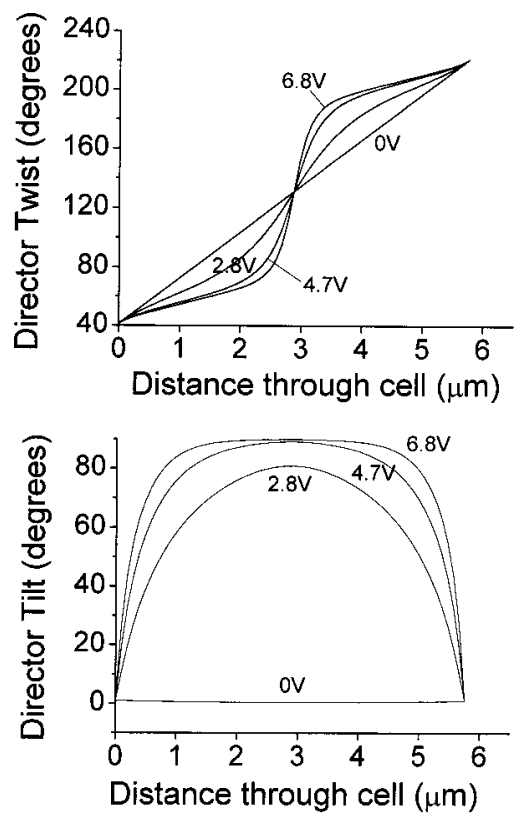

FIG. 3. Director profiles for different applied voltages: 0, 2.8, 4.7, and $6.8 \mathrm{~V}$.

elastic constants, the dielectric and optical permittivities, the director surface angles, and the cell thickness, are set from the fitting just described. By choosing suitable viscosity coefficients, the theoretical results obtained from the program DIMOS are compared with the dynamical experimental data at different times during the switching process. Figure 4 shows the fits of the guided-mode data, $T_{p s}$ and $T_{s s}$, at different times during switch-off from $4.7 \mathrm{~V}$. Values for the viscosity coefficients $\gamma, \eta_{1}, \eta_{2}, \eta_{3}$, and $\eta_{12}$ are $(0.120 \pm 0.001)$, $(0.170 \pm 0.002), \quad(0.018 \pm 0.004), \quad(0.04 \pm 0.01), \quad$ and $(0$ $\pm 0.04)$ Pas, respectively. The rotational viscosity $\gamma$ plays the most important role, with $\eta_{1}$ also significant since most of the director in the cell is parallel to the velocity gradient of the flow (the analysis in detail can be found in Ref. 11).

From these fits, the changes of the director structure in the cell during the switch-off process are obtained. They are shown in Fig. 5 for different times after removal of $4.7 \mathrm{~V}$ from the cell. It is found that at the beginning of the switchoff, the director twist near the cell surfaces decreases. This means that, comparing with the director twist state in the longer time after the switch-off, the director twist near the cell surface is in the reverse direction from the $0 \mathrm{~ms}$ state and closer to the easy axis of the surface, making more surface part of the cell in the nearly same director twist. However, unlike in the TN cell, this reversal of the director twist does not go over the direction of director twist on the surface. After about $5 \mathrm{~ms}$, the director twist recovers back to its initial state, towards linearity. The more important point from Fig. 5 is that the director tilt in the cell center is only decreases monotonically during the switch-off process, never going beyond the initial tilt angle even at the early stages of the dynamic process. This means that there appears to be no backflow in the cell or, if there is, it is of such a character that it is insufficient to drive the tilt beyond homeotropic in the STN cell after a $4.7 \mathrm{~V}$ electric field is switched off. This 

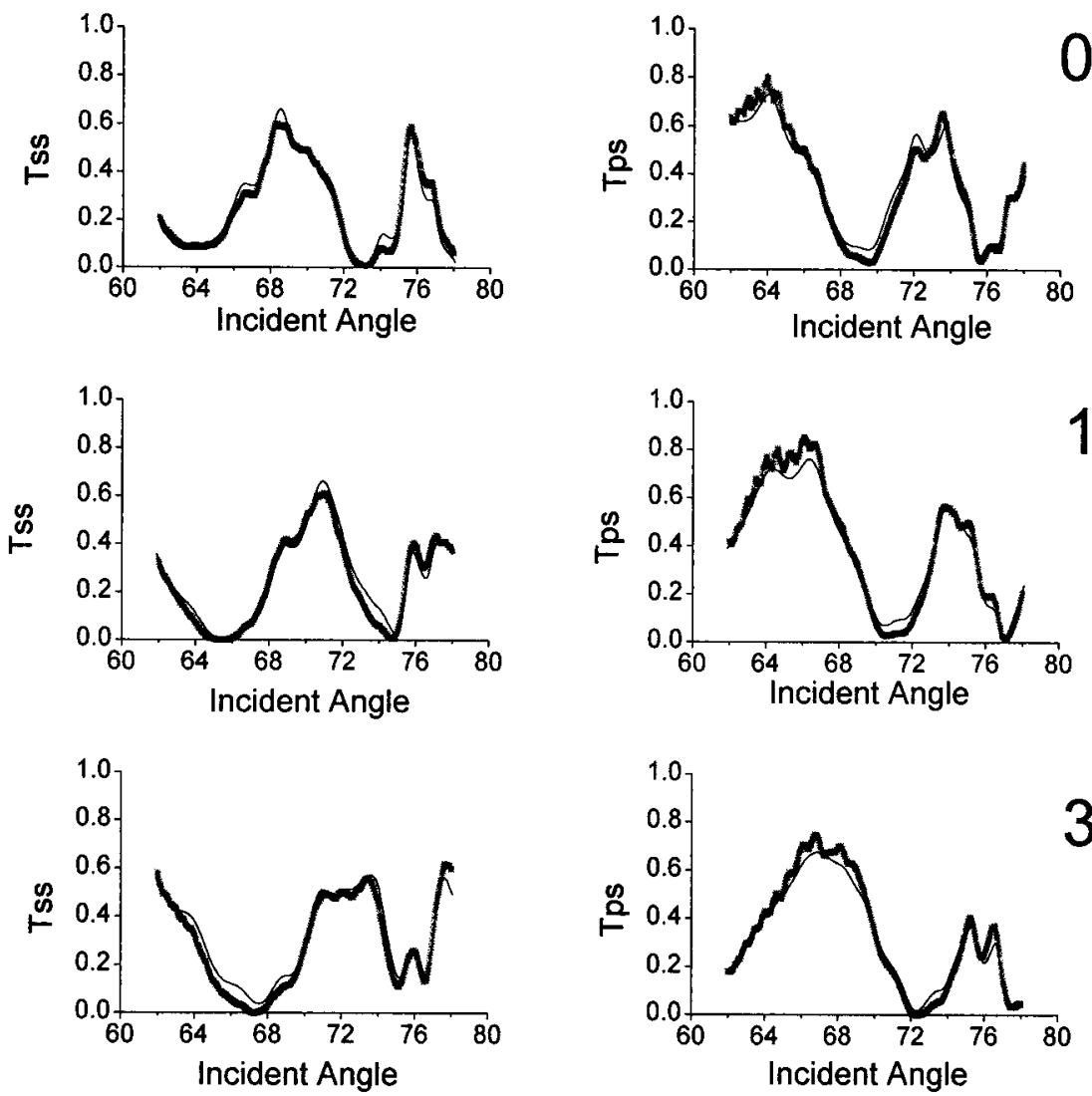

$3 \mathrm{~ms}$
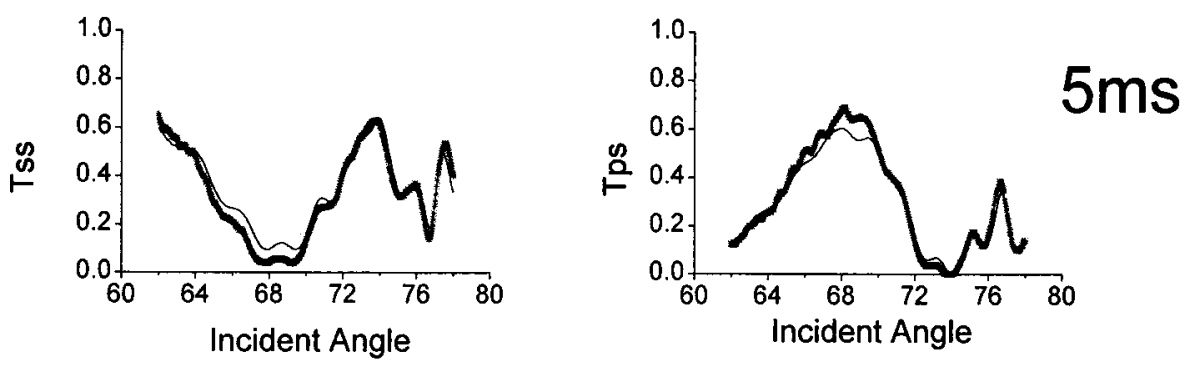

FIG. 4. Dynamic data $T_{s s}, T_{p s}$ (crosses) with the theoretical fits (solid line) at different times after switch-off of $4.7 \mathrm{~V}$ ac from the cell.
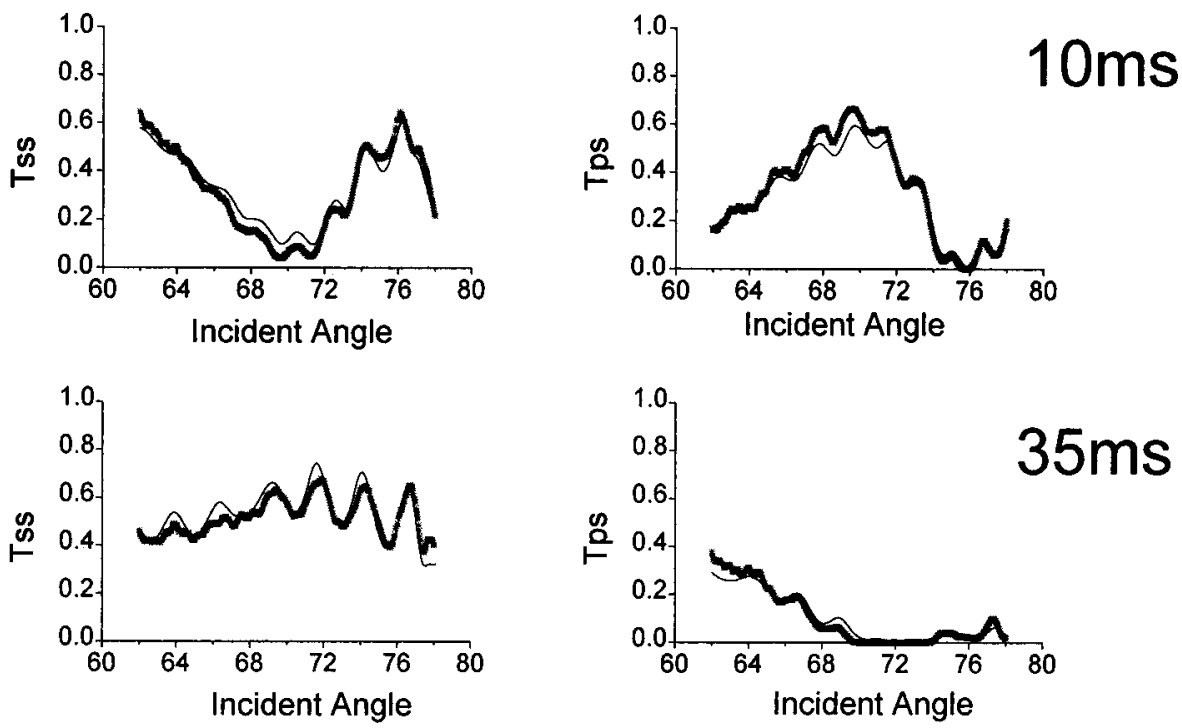

is the most significant difference between the STN cell and the TN cell, where the backflow causes a maximum director tilt beyond $90^{\circ}$ in the cell center at the beginning of the switch-off.
Consider this situation analytically. Suppose the initial director profile near the cell surfaces for $4.7 \mathrm{~V}$, as shown in Fig. 6(a). The director twists through $180^{\circ}$ and the directors near the two surfaces are nearly in the same plane, which is 

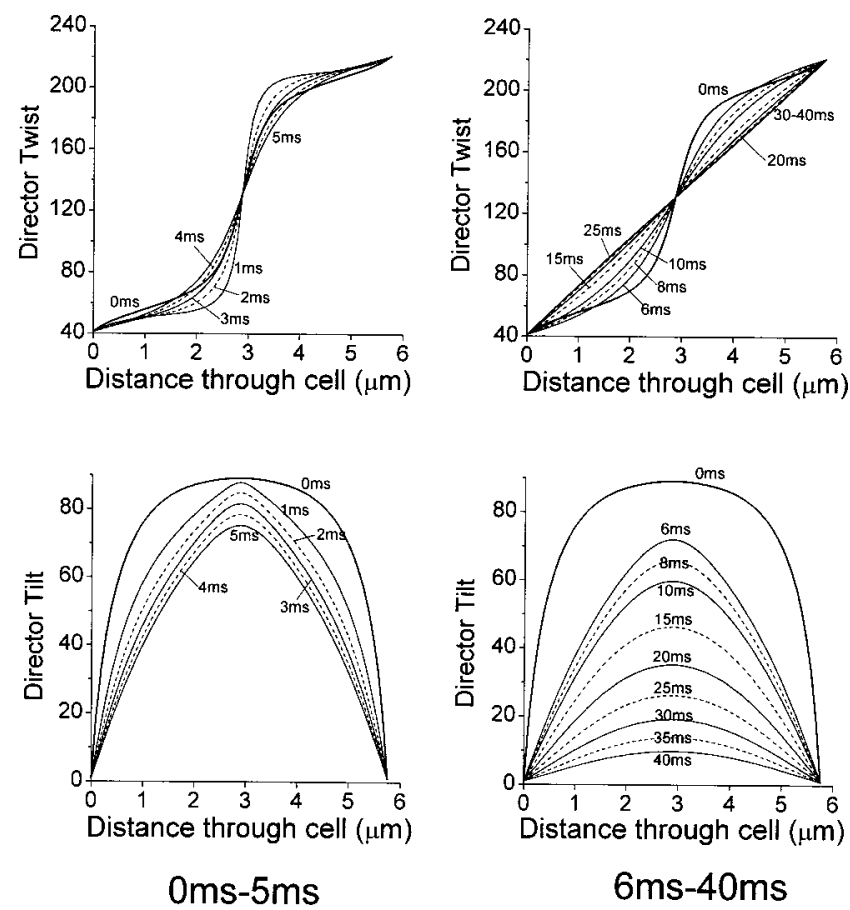

FIG. 5. Director profiles at different times after switch-off of $4.7 \mathrm{~V}$ ac from the cell.

the $X-Z$ plane in Fig. 6(a). From Fig. 3, the elastic torque (balanced by the field torque) will be greatest at about $Z$ $= \pm(1 / 5) d$ (cell thickness), where the second spatial derivative of the tilt is greatest. When the field is switched off, the unbalanced elastic torque causes a clockwise rotation of the director in the bottom region and an anticlockwise rotation of the director in the top region [shown in Fig. 6(a) by the curved arrows]. Because of coupling between director rotation and hydrodynamic motion, fluid flow is induced, as shown by the long arrows. From Fig. 6(a), it is clear that this fluid motion near the top and bottom of the cell has the same direction and this does not cause any torque to act on the director in the middle region of the cell, as happens in the $\mathrm{TN}$ cell. Thus, after the electric field switches off from the

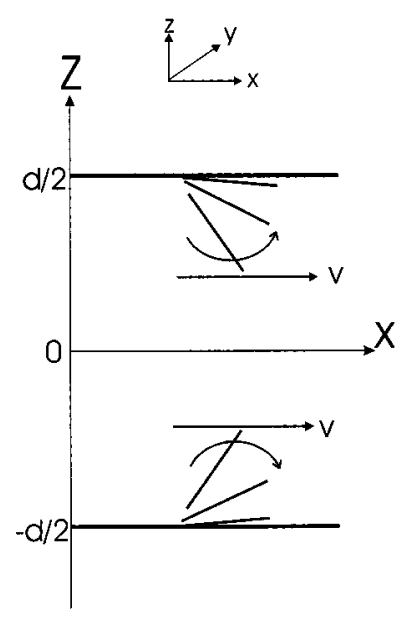

(a)

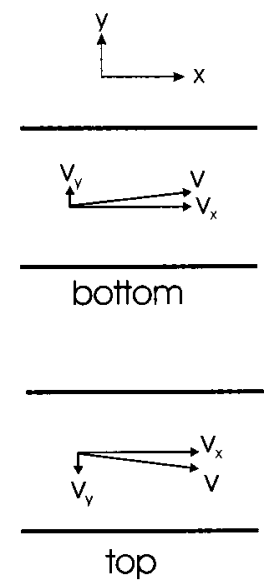

(b)
FIG. 6. The hydrodynamic analysis for no-backflow switch-off process. cell, the director tilt in the center of the cell will simply decrease continuously, thus, while there is certainly induced flow, there is no backflow. This analysis explains the experimental result. In addition, if one further considers the small deviation of the director twist from the easy axis (here the $X$ axis) at around $Z= \pm(1 / 5) d$, the rotation of the director at the beginning of the switch-off would induce a small additional component of fluid flow along the $Y$ axis, as shown in Fig. 6(b). Now, the director twist at the cell center in the initial state is along the $Y$ axis in the STN cell. Thus, a small torque in the middle region of the cell, produced by the flow along the $Y$ axis, would be expected to act on the director in this region. In general, however, because the deviation of the director twist near the surfaces is very small, this flow torque is also very small. With suitable choice of material, this torque does not play a significant part in the change of the director tilt during the dynamic process, as found here. However, if the applied electric field is very high and the rotational viscosity of the filling material is very large, this torque may give rise to an increase of the director tilt in the middle of the cell at the beginning of switch-off, that is, a weak backflow may be introduced. Therefore, as is well known, the choice of the filling material is very important for fast STN cells.

\section{CONCLUSIONS}

Using the convergent-beam system together with the fully leaky guided-mode technique, the director relaxation of a $180^{\circ}$ STN cell, filled with chiral LC has been studied in detail, after removal of an ac field. It is shown that the experiment results fit very well with the approximation of Berreman and van Doorn of the Ericksen-Leslie theory.

The relaxation time of the switch-off process, which is much shorter than that in a TN cell, depends primarily on the cell parameters, being largely independent of the initial applied voltage. The director profiles at different times during the switch-off have been obtained. At the beginning of the relaxation, the director twist near the cell surface tends towards the easy axis of the surface. There is no significant backflow for the director tilt in this $180^{\circ} \mathrm{STN}$ cell. The fluid flow state in the cell at the early stages of the switch-off has been analyzed to help understand this no-backflow dynamic process.

\section{ACKNOWLEDGMENTS}

This work was conducted under both the project no. 10174044 supported by NSFC and the Royal Society Joint UK-China Research Grant.

${ }^{1}$ J. L. Ericksen, Arch. Ration. Mech. Anal. 4, 231 (1960).

${ }^{2}$ J. L. Ericksen, Trans. Soc. Rheol. 5, 23 (1961).

${ }^{3}$ F. M. Leslie, Q. J. Mech. Appl. Math. 19, 357 (1966); Arch. Ration. Mech. Anal. 28, 265 (1968); Advances in Liquid Crystals, edited by G. H. Brown (Academic, New York, 1979), Vol. 4, p. 1.

${ }^{4}$ D. W. Berreman, J. Appl. Phys. 46, 3746 (1975).

${ }^{5}$ C. Z. van Doorn, J. Appl. Phys. 46, 3738 (1975).

${ }^{6}$ C. J. Gerritsma, C. Z. van Doorn, and P. van Zanten, Phys. Lett. 48A, 263 (1974). 
${ }^{7}$ F. Nakano, H. Kawakami, H. Morishita, and M. Sato, Jpn. J. Appl. Phys. 19, 659 (1980).

${ }^{8}$ O. Cossalter and D. A. Mlynski, Liq. Cryst. 19, 545 (1995).

${ }^{9}$ N. J. Smith and J. R. Sambles, J. Appl. Phys. 85, 3984 (1999).

${ }^{10}$ N. J. Smith and J. R. Sambles, Appl. Phys. Lett. 77, 2632 (2000).

${ }^{11}$ L. Ruan and J. R. Sambles, J. Appl. Phys. 92, 4857 (2002).
${ }^{12}$ L. Ruan and J. R. Sambles, Phys. Rev. Lett. 90, 168701-1 (2003).

${ }^{13}$ S. J. Elston and J. R. Sambles, Liq. Cryst. 200, 167 (1991).

${ }^{14}$ F. Yang and J. R. Sambles, J. Opt. Soc. Am. B 10, 858 (1993).

${ }^{15}$ L. Ruan, J. R. Sambles, and J. Seaver, Liq. Cryst. 19, 133 (1995).

${ }^{16}$ F. Yang and J. R. Sambles, J. Opt. Soc. Am. B 16, 488 (1999).

${ }^{17}$ D. Y. K. Ko and J. R. Sambles, J. Opt. Soc. Am. A 5, 1863 (1988). 